\section{Military Technical College \\ Kobry El-Kobbah, Cairo, Egypt.}

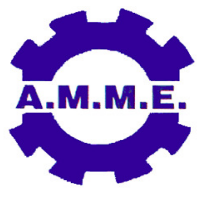

\title{
EXPERIMENTAL STUDIES ON \\ HYDRODYNAMIC CHARACTERISTICS OF A VERTICAL PLUNGING LIQUID JET SYSTEM
}

\author{
A. K. Roy , B. Maiti* and P. K. Das ${ }^{* *}$
}

\begin{abstract}
The present investigation is mainly concerned with experimental studies on the hydrodynamic characteristics of vertical plunging liquid jet, impinging on a liquid surface. To study the structure of biphasic zone below the free surface of liquid, needle type electrical conductivity probe has been designed and extensively used for the present study. To visualize the two-phase flow field, a high-speed digital camera is also used. The present study provides new methods and application of phase detection probe to understand the physics of the plunging jet aeration system. Apart from the air entrainment characteristics, the geometry of the biphasic zone for the vertical plunging liquid jets also has been investigated. The experimental results are also compared with the existing correlations and are found to be in good agreement. The overall study contributes to the better understanding of air entrainment process and bubble dispersion for the vertical plunging liquid jets.
\end{abstract}

\section{KEY WORDS}

Air entrainment, vertical plunging jet, flow visualization, conductivity probes.

\section{NOMENCLATURE}

dj Jet diameter at the impact point, $\mathrm{mm}$.

$\mathrm{d}_{\mathrm{N}} \quad$ Diameter of nozzle, $\mathrm{mm}$.

$\mathrm{F}_{\mathrm{B}} \quad$ Bubble count rate, $\mathrm{s}^{-1}$.

$\mathrm{Fr}$ Froude number, dimensionless.

$\mathrm{H}_{\mathrm{N}}$ Height of nozzle from free surface of liquid, mm.

$\mathrm{H}_{\mathrm{P}} \quad$ Maximum bubble penetration depth, $\mathrm{mm}$.

$L_{i} \quad$ Jet length, $m m$.

$\mathrm{L}_{\mathrm{N}} \quad$ Nozzle length, $\mathrm{mm}$.

\footnotetext{
* Associate professor, Dpt. of Mechanical Engineering, B I T Mesra, India.

** Professor, Dpt. of Mechanical Engineering, I I T Kharagpur, India.
} 
$Q_{G} \quad$ Volumetric flow rate of gas or air, $\mathrm{m}^{3} / \mathrm{s}$.

$Q_{L} \quad$ Volumetric flow rate of liquid or water, $\mathrm{m}^{3} / \mathrm{s}$.

$r$ Radial distance, $\mathrm{mm}$.

$\mathrm{V}$ Probe signal, Volt.

$\mathrm{V}_{\mathrm{C}}$ Inception velocity, $\mathrm{m} / \mathrm{s}$.

$V_{j} \quad$ Jet velocity at the impact point, $\mathrm{m} / \mathrm{s}$.

$V_{N} \quad$ Jet velocity at the exit of the nozzle, $\mathrm{m} / \mathrm{s}$.

$\mathrm{V}^{*} \quad$ Normalized probe signal, dimensionless.

$\mathrm{z} \quad$ Vertical or axial distance, $\mathrm{mm}$.

\section{INTRODUCTION}

Liquid jets impinging on the free surface of a liquid pool are frequently encountered in the nature, in many industrial as well as in man made situations. The most spectacular observation regarding the plunging liquid jet is the entrainment of air bubbles below the free surface of liquid. Plunging liquid jet is a moving liquid column that passes through a gaseous space and impinges on the free surface of another liquid pool. Plunging liquid jets have significant role in dispersion of air bubbles into water bodies in natural processes of self-purification of rivers; streams; waterfalls and weirs. In oceans, the top of the wave front is a water jet; which entrains large amount of air bubbles when it breaks over the free surface of water ahead of it.

The phenomenon of air entrainment due to plunging liquid jets is complex in nature. A jet of liquid when strikes on another liquid body at a low velocity, it results in formation of small depression on the free surface of that liquid. With the increase in the jet velocity, the depression on the free surface of the liquid deepens and an air sheath or envelop is formed on the periphery of the liquid jet at the impact point just below the free surface of liquid. This air sheath or envelop is very unstable in nature. The formation and collapse of the air sheath induces air bubbles below the liquid surface. Thus, atmospheric air is entrained in the liquid body.

The entrained air bubbles reach up to a certain depth below the liquid surface, and then start rising in the upward direction in a helical path. The upward rising bubbles form a concentric envelop around the downward biphasic cone.

Air entrainment due to plunging liquid jet has many advantages. Plunging jet aeration may be used effectively in dissolving air in some gas-liquid reactors. In biological processes, spargers or airlift fermenters are extensively used for aeration along with jet aeration. The jet aerators have potential application in many chemical fermentation and floatation processes, due to it's simple construction and relatively less operational difficulties.

The most interesting observation regarding the plunging liquid jet is the entrainment of air bubbles below the surface of liquid and formation of biphasic zone. The mechanism of air entrainment due to plunging liquid jets is quite complex. Before 1970 , there were only a few serious attempts made to study the phenomena of air entrainment by plunging liquid jets. Since then, the interest and the scope of the experimental study on plunging liquid jet systems have increased. 
Bin and Smith[1], Bin[2] reviewed the various aspects of plunging liquid jet system. Cummings and Chanson[3] observed that air entrainment due to plunging jets can only takes place when the liquid jet impact velocity exceeds a certain critical velocity which is related to jet inflow conditions.

Observation made by Robertson et. al.[4] and Michel[5] showed that the formation of a stable air sheath around the jet depends on the ambient pressure and the air sheath behaves as a ventilated cavity. There is considerable fluctuation in the length of the air sheath and at the lower end of air sheath air pockets are entrained by discontinuous 'gusts'.

The measurement of the length of the air sheath was carried out by Kusabiraki et. al.[6] using high-speed photographic technique. They have reported that with the increase in liquid jet velocity the length of the air sheath increases.

The classification of the mechanism of air entrainment was reported by McKeogh and Ervine[7]. Davoust et. al. [8] observed that the air entrainment process due to plunging liquid jet as the consequence of the interfacial shear at the surface of the liquid jet which drags and entraps the air boundary layer at the point of impact of the plunging jet with the receiving pool. The studies on inception condition for different range of Reynolds number and Weber number of liquid jet has been reported by El Hummoumi et. al.[9].

The experimental studies on basic entrainment characteristics in air-water plunging liquid jet system for a wide range of flow situations were carried out by Chanson et. al.[10-11].They have observed the lowest inception velocity as $0.73 \mathrm{~m} / \mathrm{s}$ in the entire range of their experimental condition. They have also observed that for liquid jet velocities slightly greater than the inception velocity, entrainment of individual air bubbles occur. At larger velocities air cavity develops around the jet and most of the air is entrained by elongation, stretching and breakup of the ventilated cavity. It has been as observed that for jet velocities slightly greater than inception velocity, the entrained air bubbles having sizes of around $0.5-1 \mathrm{~mm}$ and for larger jet velocities the sizes of the entrained bubbles in the developing flow region are in the range of $0.5-5 \mathrm{~mm}$.

Chanson et. al.[12] carried out experiments to understand bubble entrainment and dispersion in plunging jet flows using fresh water and sea water. The characterization of plunging liquid jets using a combined experimental and numerical technique has been carried out by Qu et. al.[13].

It is observed that the variables like jet diameter, jet velocity, jet length and physical properties of the fluid affect the entrainment of air due to plunging liquid jet. Literature review reveals that observations of different investigators are not always similar in nature; therefore, further investigations are required to gather an insight into the characteristics of air entrainment through plunging liquid jet.

\section{EXPERIMENTATION}

The experimental setup fabricated for the present study is shown in Fig.1. More detail of the experimental setup can be found in Roy et. al.[14]. However, for better 
understanding, some of the relevant features are described below from Roy et. al.[14]. The setup consists of the following major components.

(i) Transparent Acrylic Tank: The tank is fabricated from $10 \mathrm{~mm}$ transparent acrylic sheets. The dimension of the tank is $1500 \mathrm{~mm}$ in length, $750 \mathrm{~mm}$ in width and $750 \mathrm{~mm}$ in height. Near the bottom of the side vertical faces two $20 \mathrm{~mm}$ openings are provided. These two openings are connected to the suction side of a centrifugal pump. This facilitates circulation of water in the experimental system.

(ii) The nozzle for jet impingement: For the purpose of jet impingement on the surface of water in the tank, two different nozzles are used. The inner diameters of the nozzles are $4 \mathrm{~mm}$ and $6 \mathrm{~mm}$ respectively. The length of those nozzles is kept constant and is $200 \mathrm{~mm}$. The nozzle is feed with water from the delivery of centrifugal pump. A pre-calibrated orifice meter fitted in line measures the flow rate through the nozzle.

(iii) Traversing mechanism for the nozzle: For the purpose of the experimentation, the location of the nozzle outlet can be varied through a traversing mechanism on to which the nozzle is attached. Graduated scales placed on the traversing mechanism can measure the position of the nozzle in the horizontal plane. The nozzle can also move in the vertical direction so that the distance between the nozzle outlet and water surface can be varied.

(iv) Flow visualization system: To visualize the nature of the two-phase flow a digital camera (SONY DSC-F717, Sony Electronics Inc. NJ) is used. The images were analyzed frame wise to visualize the geometry of the biphasic zone and to understand two-phase flow phenomenon due to liquid jet impingement.

(v) Phase detection probe: To study the hydrodynamic characteristics of the twophase flow, a needle type single tip conductivity probe has been designed and fabricated for the present purpose as shown schematically in Fig.2. The probesensing element is primarily a fine stainless steel wire of $0.06 \mathrm{~mm}$ in diameter having a length of $4 \mathrm{~mm}$. The wire is coated with a fine layer of epoxy resin and is connected with the signal cable. However, care has been taken to ensure that only the tip of the sensing wire is exposed. The signal cable passed through an 'L' shaped insulated stainless tube of $6 \mathrm{~mm}$ diameter. Thus ' $L$ ' shaped tube provides support to the sensing element. The sensing wire is the primary electrode where as the second electrode is in the form of a copper plate placed away from the primary electrode dipped inside the tank. The probe is connected with a suitable traversing mechanism. Before using the probe, it is properly calibrated to detect the gas and liquid phases. The principle of operation of conductivity probe is based on electrical conductivity of the medium. When the medium is only liquid between, the electrical resistance between two pairs of electrodes is the least. Therefore, the voltage of electrical signal obtained over a time period will be constant and this has been calibrated and set to a nondimensional value of one. Whenever, presence of gas phase appears between the said pair of electrodes, the overall electrical resistance will increase and nondimensional value of voltage will be less than one. This adopted principle is 
clearly visible in Fig.9, where, signals for liquid phase and different gas-liquid phases have been reported.

\section{Experimental Procedure}

The pump which receives water from the tank again discharges into the water surface through the nozzle [14]. The flow rate through the nozzle can be controlled and monitored. During experimentation, whenever the temperature of water raised by approximately $2^{0} \mathrm{C}$, the experimentation was discontinued till the water temperature falls back to initial temperature. The temperature of water during the experimentation is around $20{ }^{\circ} \mathrm{C}$. The range for which experimentation is conducted is given in Table 1.

\section{RESULTS AND DISCUSSION}

\section{Visual and Photographic Observation}

For the sake of continuity some of the important observations are included in this section from [14]. A schematic representation of air entrainment and bubble formation below the free surface of water is shown in Fig.3. The inception velocity for which air entrainment is observed is given in the Table 2.

When the jet velocity is just above the inception velocity, air entrainment takes place in the form of few individual small bubbles; where entrainment is intermittent in nature. With the increase in jet velocity, continuous entrainment of air is observed. The downward bubble velocity decreases gradually to zero and it starts rising towards the free surface of the liquid. Some bubbles may reach at the surface, while other bubbles are carried downwards with the bubbles moving down and continue with a circulatory motion in the vertical plane, prominently near the edge of the biphasic cone. A typical biphasic zone is shown in Fig.4. In the present study, the visual observation of the phenomenon of air entrainment agrees with the observations of Bin[2], Mckeogh and Ervine[7], Chanson et. al.[11].

When the nozzle velocity is lower compared to the earlier situation, typical vortex formation is observed from the biphasic cone as shown in Fig.5. This observation of vortex formation is also reported by Bonetto and Lahey[15].

\section{Geometry of the Biphasic Cone}

From the visual observations and analysis of the photographs taken within the range of experimentation for both $4 \mathrm{~mm}$ and $6 \mathrm{~mm}$ nozzles, the angle of the biphasic cone is found to be in the range of 11.36 to 13.2 degrees. This biphasic cone angle is also similar to the value obtained from the measurement by traversing the probe within the biphasic zone in the radial direction at various depths from the free surface of water. The present result is in good agreement with the result obtained by Chanson et al. [11]. Chanson et al. observed the average angle of the biphasic cone as 13 degrees. 


\section{Bubble Penetration Depth}

At the maximum penetration depth, the bubble velocity reduces to zero so, due to the effect of buoyancy, the bubbles rise freely up to the free surface of liquid. Estimation of penetration depth is crucial for the performance of a plunging liquid jet system. The bubble penetration depth is measured with the help of a digital camera and using a graduated scale attached into the tank. The present experimental data is compared with the existing correlations and are given below.

Suciu and Smigelschi[16]

$\mathrm{H}_{\mathrm{P}}=10\left(\mathrm{~d}_{\mathrm{N}} * \mathrm{v}_{\mathrm{N}}\right)$

(valid for the range $v_{N}: 2.2-9.7 \mathrm{~m} / \mathrm{s}, \mathrm{d}_{N}: 1 \times 10^{-3}-4 \times 10^{-3} \mathrm{~m}, \mathrm{~L}_{\mathrm{j}} / \mathrm{d}_{\mathrm{N}}>20$ )

McKeogh and Ervine[7]

$\mathrm{H}_{\mathrm{p}}=2.6\left(\mathrm{~d}_{\mathrm{j}} * \mathrm{v}_{\mathrm{j}}\right)^{0.7}$

(valid for the range of $\mathrm{v}_{\mathrm{N}}: 1-10 \mathrm{~m} / \mathrm{s}, \mathrm{d}_{\mathrm{N}}: 6 \times 10^{-3}-2.5 \times 10^{-2} \mathrm{~m}$ )

Van de Donk[17]

$\mathrm{H}_{\mathrm{P}}=2.4\left(\mathrm{~d}_{\mathrm{j}} * \mathrm{~V}_{\mathrm{j}}\right)^{0.66}$

(valid for the range of $v_{N}: 3-13 \mathrm{~m} / \mathrm{s}, \mathrm{d}_{N}: 4 \times 10^{-3}-4 \times 10^{-1} \mathrm{~m}, \mathrm{~L}_{\mathrm{j}} / \mathrm{d}_{\mathrm{N}}: 3.5-50$ )

The results of the present experimental data along with the existing correlations for 4 $\mathrm{mm}$ nozzle and $6 \mathrm{~mm}$ nozzles are shown in Figs. 6-7, respectively. It is observed that the bubble penetration depth increases with the increase in jet velocity and decreases with the increase in nozzle height. This agrees with the observations of the above researchers. It is observed that the data for $4 \mathrm{~mm}$ nozzle are in close agreement with the correlation by McKeogh and Ervine [7] at $\mathrm{H}_{\mathrm{N}}=0.300 \mathrm{~m}$, whereas at $\mathrm{H}_{\mathrm{N}}=0.050 \mathrm{~m}$, correlation by Suciu and Smigelschi [16] agrees somewhat better than the other two correlations. The correlation by Van de Donk [17] under predicts the data of the present experimental results for $4 \mathrm{~mm}$ nozzle. In the case of $d_{N}=6$ $\mathrm{mm}$, at $\mathrm{H}_{\mathrm{N}}=0.300 \mathrm{~m}$, correlation by Van de Donk [17] agrees very well with the present data, where as at $\mathrm{H}_{\mathrm{N}}=0.050 \mathrm{~m}$, the data points are found to lie in between the correlations by McKeogh and Ervine [7] and Suciu and Smigelschi [16]. Here it is to be noted that due to limited height of the nozzle from the free surface of water, the velocity of jet at the impact point $\left(v_{j}\right)$ has been taken equal to the jet velocity at the nozzle exit $\left(v_{N}\right)$ and diameter of the jet at impact $(\mathrm{dj})$ is equal to the inner diameter of the nozzle $\left(d_{N}\right)$ for the entire range of experimentation.

\section{Trajectory of a Single Bubble}

The trajectory of a single bubble entrained due to liquid jet impingement could be obtained by entrainment of a single air bubble and by noting its coordinates over a time period from photo images. This is shown in Fig.8. The random movement of the bubble suggests that why jet aerators have good mixing characteristics in gas-liquid reactors.

\section{STRUCTURE OF TWO-PHASE FLOW}

To understand the structure of two-phase flow, the probe tip is traversed in radial and axial direction to capture the probe signals which are recorded continuously. 
Analyzing the normalized probe signals and their Probability Distribution Functions (PDFs) the flow structure is identified.

\section{Probe Signals in Axial Direction}

The probe signals, Probability Density Function (PDF) curves and the time delay plots for parameters, velocity of water at nozzle exit $\left(\mathrm{v}_{\mathrm{N}}\right)=3.32 \mathrm{~m} / \mathrm{s}$, diameter of the jet $\left(d_{N}\right)=6 \mathrm{~mm}$, height of the nozzle from free surface of water $\left(\mathrm{H}_{\mathrm{N}}\right)=300 \mathrm{~mm}$ at four different normalized axial positions $\left(z / H_{P}\right)=0.1,0.4,0.8$ and 1.0 below the free surface of water along with the liquid phase signal are shown in Figs. 9(I-V). A time series consists of a set of observations, which is made subsequently in time scale. Sets of data for time series plot can be generated by equidistantly sampling of continuous-time signal $\mathrm{V}(\mathrm{t})$ at a constant sampling rate. The time series, as we know, basically is a record showing how a quantity changes with time. However, importance lies in the fact that, during visual observation, one can get important information on the trends of a signal acquired for a long duration, periodicities, randomness, rare events, missing points etc. In view of the usefulness of the time delay plot, the experimental results have been plotted in terms of $\mathrm{V}(\mathrm{t}+2 \tau)$ versus $\mathrm{V}(\mathrm{t})$. Here $\tau$ represent the time step delay. The delay plot is expected to give an insight into the differences occurring in two-phase flows and the flow regime identifications.

Figs. 9 (la-lc)) show the normalized probe signal, PDF plot and the time delay plot for the liquid (water) phase alone. Fig. 9 (la) is basically showing the noise in the liquid phase signal. Fig. 9 (lb) is the corresponding PDF curve for the liquid phase signal. The width of the base of the PDF signal is very narrow. The width is nearly is the same as the width of the noise in the liquid phase signal. The attractor of the time delay plot is concentrated near coordinate $(1,1)$ to a very narrow zone as shown in Fig. 9 (IC). As entrainment of air due to plunging liquid jets takes place, the sensing tip of the conductivity probe get in contact with the gas phase bubbles. So, undulations in the probe signal appear. Figs. 9 (Ila-Ilc) show the normalized probe signal, PDF curve and time delay plot at $\mathrm{z} / \mathrm{H}_{\mathrm{P}}=0.1$ foe $\mathrm{v}_{\mathrm{N}}=3.32 \mathrm{~m} / \mathrm{s}$. The probe signals show the undulations are smaller in amplitude but are large in number. This represents the presence of smaller bubbles in large number. This is also depicted in the PDF curve as shown in Fig. 9 (Ilb). The base of the PDF curve is little wider than for the pure liquid phase PDF curve. The attractor in the time delay plot also shows the existence of liquid phase noise and it extends the origin along a line approximately $45^{0}$ to both the ordinates. The extension of the origin in the time delay plot depends on the drop in the voltage signal and the nature of undulations. It has been observed that the less the diameter of the bubbles, the less is the voltage drop in the probe signal. Here the upper portion of the attractor in the time delay plot is observed to be denser than the lower portion. The dense nature of the attractor near the coordinate $(1,1)$ in the time delay plot represents the presence of large number of tiny bubbles in the two-phase flow. With the increase in $\mathrm{z} / \mathrm{H}_{\mathrm{p}}$, coalescence of the air bubbles take place, so the probe signals show larger undulations as shown in Figs. 9(III-IV). The base of the PDF curve also widens and the height of the PDF curve decreases. The attractor in the time delay plot also extends more nearer to the origin of the ordinates and the attractor become less dense near the coordinate $(1,1)$, thus represents the existence of relatively larger bubbles. 


\section{Probe Signals in Radial Direction}

The probe signals, PFD curves and the time delay plots for $\mathrm{v}_{\mathrm{N}}=3.32 \mathrm{~m} / \mathrm{s}, \mathrm{d}_{\mathrm{N}}=6$ $\mathrm{mm}, \mathrm{H}_{\mathrm{N}}=300 \mathrm{~mm}$, and at $\mathrm{z} / \mathrm{H}_{\mathrm{P}}=0.25$ at four different radial positions $(r), 0 \mathrm{~mm}, 4$ $\mathrm{mm}, 14 \mathrm{~mm}$ and $44 \mathrm{~mm}$ respectively are shown in Figs. 10(I-IV). From the Figs. $10(\mathrm{I}-\mathrm{IV})$, the probe signals show some gross nature of bubbly flow pattern at various radial positions. Near the centerline of the biphasic zone, the probe sensor interacts with the downward smaller primary bubbles. The base of the PDF curve is observed to be less wide for probe signals near the centerline of the biphasic zone than away from the centerline. The attractor in the time delay plot is also extends less in distance towards the origin of the ordinates. As the probe is traversed radially away from the centerline of the biphasic zone, the recorded signals show the existence of relatively larger bubbles so local shift of the signals are observed. The base of the PDF curve becomes wider as shown in Fig. 10(II). Similarly the attractor in the time delay plot also observed to be different in length along a $45^{\circ}$ line to both the ordinates. At $r=14 \mathrm{~mm}$ the probe is in contact with dispersed upward secondary bubbles. So a sudden change in the shape of the PDF curves is observed, as shown in Fig. 10(III). So, the base of the PDF curve again narrows down and height of the PDF curve increases. This shows that the probe tip is more in contact with water than air. The decrease in the length of the attractors is also observed. In highly dispersed bubbly flow region at $r=44 \mathrm{~mm}$, the attractor shows a typical shape as shown in Fig. 10(IV).

\section{Bubble Count Rate}

To understand the nature of bubble count rate within the entire two phase flow zone, a detailed study has been made using single tip conductivity probe. To measure the nature of bubble count rate the probe is traversed from jet centerline up to a point where the traces of bubbles are seen in radial direction. The bubble count rate is estimated from the raw probe signals using software developed for this purpose. The probe is positioned at different depths $\left(z / H_{P}=0.25-0.75\right.$ ) below the free surface of water. A typical variation of bubble count rate along radial direction for $\mathrm{v}_{\mathrm{N}}=5.50 \mathrm{~m} / \mathrm{s}$ at different depths are shown in Figs. 11(a-c). From the Figs.11(a-c), some distinct peak and off-peak points are observed. The relative positions of peak and off-peak of $F_{B}$ profiles within the flow field are shown schematically in Fig.12. In Fig. 11(a) at $r=0 \mathrm{~mm}$, the bubble count rate starts from some value and then at a certain radial distance from the axis of the jet, the $F_{B}$ profile show a peak, represented as first peak of $F_{B}$. Then there is a sharp fall in the value of $F_{B}$ up to a certain radial position, represented as first off-peak of $F_{B}$. The value of $F_{B}$ again increases up to a certain radial distance from jet centerline represented as second peak of $F_{B}$. Finally the $F_{B}$ decreases with increase in radial distance. From Fig. 11(a), the first peak of $F_{B}$ is found at a radial distance $r=3 \mathrm{~mm}$, which is near to the edge of the jet. This agrees with the observations by Chanson et. al.[11]. They argued that the maximum bubble count rate occur at the inner shear region, which is very near to the edge of the submerged jet. The first peak of $F_{B}$ is at a radial distance near the jet edge, whereas the first off-peak of $F_{B}$ is near the edge of the biphasic cone. The radial distance between the first peak of $F_{B}$ and first off-peak of $F_{B}$ represents the recirculation zone. A similar trend is found for other depths as shown in Figs. 11(b-c). 


\section{Probe Signals within the Air Sheath}

To understand the nature of probe signals within the air sheath and away from it, the probe is traversed from $r=0 \mathrm{~mm}$ to $\mathrm{r}=5 \mathrm{~mm}$ in the step of $1 \mathrm{~mm}$ for $v_{\mathrm{N}}=5.50 \mathrm{~m} / \mathrm{s}$, $\mathrm{d}_{\mathrm{N}}=4 \mathrm{~mm}, \mathrm{H}_{\mathrm{N}}=300 \mathrm{~mm}$ at $\mathrm{z}=8 \mathrm{~mm}$ below the water surface. The raw probe signals are shown in Fig.13. At $r=0 \mathrm{~mm}$ and $1 \mathrm{~mm}$, the probe signals show the presence of liquid phase with little trace of very fine bubbles. At $r=2 \mathrm{~mm}$, i.e. at the edge of the jet surface the probe tip is partial in contact with air sheath and the liquid jet. So, at this point the nature of the probe signal starts changing and the probe signal is also observed to be mildly oscillating due to partial contact with air sheath and liquid jet. At $r=3$ and $4 \mathrm{~mm}$, the probe tip is fully in contact with air sheath so, the voltage drops due to presence of air phase in contact with the probe. The oscillations of the probe signals can be observed due to oscillating nature of the air sheath. Finally at $r=5 \mathrm{~mm}$ the probe is at the zone of secondary rising air bubbles, outside the air sheath region. At this point the probe signal represents the dispersed bubbly flow with rising bubbles of reasonably larger in size.

\section{CONCLUSIONS}

Hydrodynamic characteristic of a vertical plunging liquid jet system is investigated. An effort has been made to understand the physics of the biphasic flow phenomenon through elaborate visual and photographic observations using high speed camera as well as using a special type of phase detection probe designed for the present investigation. The salient conclusions drawn from the study are summarized below:

i) The study of air entrainment through visualization reveals that the inception of entrainment is due to the formation of an air sheath surrounding the jet just below the interface. The shape of the biphasic zone formed due to vertical impinging jet resembles a truncated cone. The measured vertex angle is found to be independent of the nozzle diameter and nozzle velocity.

ii) The bubble penetration depth depends on the jet velocity and the jet height. The present experimental data are compared with the existing correlations and are found in good agreement.

iii) The trajectory of a single bubble within the downward biphasic zone has been studied using a digital camera. The trajectory shows a zigzag up and down oscillating pattern with helical motion of the bubble around the jet axis. The structure of the biphasic zone revealed some peak and off-peak bubble frequency points.

iv) The probe signals show the bubbly flow pattern within the biphasic zone. Till now researchers have postulated about the presence of air sheath based on photographic observations. Due to presence of upward secondary bubbles in front of air sheath, the photographs are generally get disturbed. Therefore, the present phase detection probe can be effectively used for finding out geometry of air sheath along with photographic technique. The probe signals within and around the air sheath reveal some interesting observations. 


\section{REFERENCES}

[1] Bin, A. K., and Smith, J. M., "Mass Transfer in Plunging Liquid Jet Absorber," Chem. Eng. Commun., Vol. 15, pp 367-383, (1982).

[2] Bin, A. K., "Gas Entrainment by Plunging Liquid Jets," Chem. Eng. Sci., Vol. 48, pp 3585-3630,(1993).

[3] Cummings, P.D., and Chanson, H.,"An Experimental Study of Individual Air Bubble Entrainment at a Planer Plunging Jet," Chem. Eng. Research and Design, Trans. IChemE, Part A, Vol. 77(A2), pp 159-164, (1999).

[4] Robertson, D. D. C., O'Shaughnessy, D. P., and Molloy, N. A., "The Mechanism of Sheath Formation by Plunging Liquid Jets," Chem. Eng.Sci., Vol. 28, pp 1635-1636, (1973).

[5] Michel, J. M., "Some Features of Water Flows With Ventilated Cavities," Fluids Engg. Trans. ASME, Vol. 106, pp 319-326, (1984).

[6] Kusabiraki, D., Niki, H., Yamagiwa, K., and Ohkawa, A., "Gas Entrainment Rate and Flow Pattern of Vertical Plunging Liquid Jets," Can. J. Chem. Eng., Vol. 68, pp 893-903, (1990).

[7] McKeogh, E.J., and Ervine, D.A., "Air Entrainment Rate and Diffusion Pattern of Plunging Liquid Jets," Chem. Eng. Sci., Vol. 36, pp 1161-1172, (1981).

[8] Davoust, L., Achard, J. L., and El Hammoumi, M., "Air Entrainment by a Plunging Jet: The Dynamical Roughness Concept and its Estimation by a Light Absorption Technique," Int. J. of Multiphase Flow, Vol. 28, pp 15411564, (2002).

[9] El Hummoumi, M., Achard, J. L., and Davoust, L., "Measurements of Air Entrainment by Vertical Plunging Liquid Jets," Experiments in Fluids, Vol. 32, pp 624-638, (2002).

[10] Chanson, H., Aoki, S., and Hoque, A., "Similitude of Air Entrainment at Vertical Circular Plunging Jets," Proc. of ASME FEDSM'02, ASME 2002 Fluids Engineering Division Summer Meeting, Montreal, Quebec, Canada, July 14-18, Paper FEDSM 2002-31024, pp 1-6, (2002).

[11] Chanson, H., Aoki, S., and Hoque, A., "Physical Modelling and Similitude of Air Bubble Entrainment at Vertical Circular Plunging Jets," Chem. Eng. Sci., Vol. 59, pp 747-758, (2004).

[12] Chanson, H., Aoki, S., and Hoque, A., "Bubble entrainment and dispersion in plunging jet flows: fresh water vs. sea water," J of Coastal Research, Vol. 22,Issue 3, pp. 664-677, (2006).

[13] Qu, X. L. „Khezzar,L., Danciu, D., Labois, M., and Lakehal, D., "Characterization of Plunging Liquid Jets: A Combined Experimental and Numerical investigation," Int. J. Multiphase Flow, Vol. 37, No. 7, pp 722-731, (2011).

[14] Roy, A. K. Maiti, B., and Das, P.K., "Visualisation of Air Entrainment by a Plunging Jet," Procedia Engineering, Vol. 56468 - 473, pp 468 - 473,(2013).

[15] Bonetto, F., and Lahey Jr, R. T., "An Experimental Study on Air Carry under Due to a Plunging Liquid Jet," Int. J. Multiphase Flow, Vol. 19, pp 281-294, (1993).

[16] Suciu, G. D and Smigelschi, O., "Size of the Submerged Biphasic Region in Plunging Jet System," Chem. Eng. Sci., Vol. 31, pp 1217-1220, (1976).

[17] Van de Donk, J. A. C., "Water Aeration with Plunging Jets," Ph.D. thesis, Technische Hogeschool Delft, (1981) 


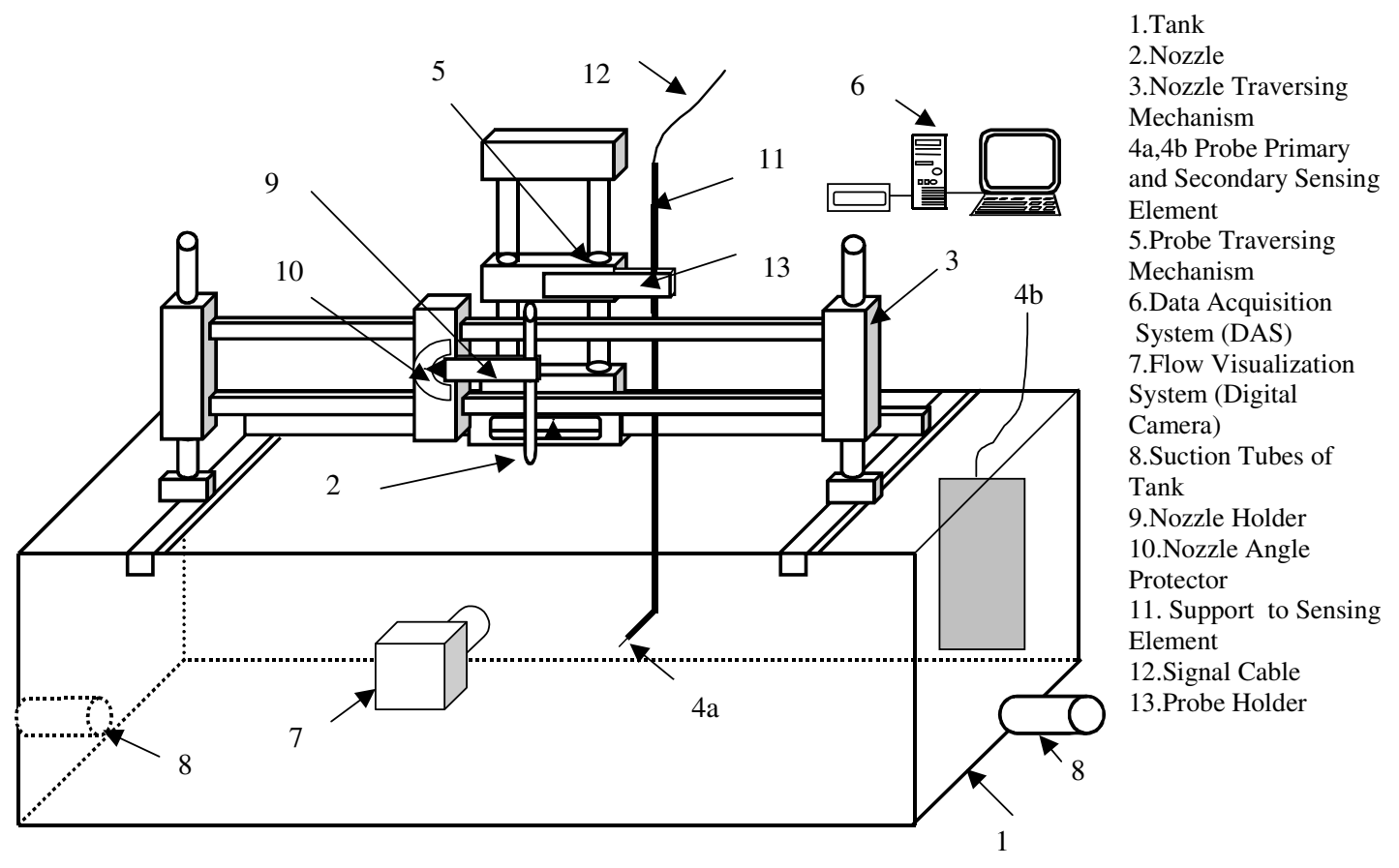

Fig. 1. Schematic diagram of the liquid jet impingement setup (Reproduced from [14])

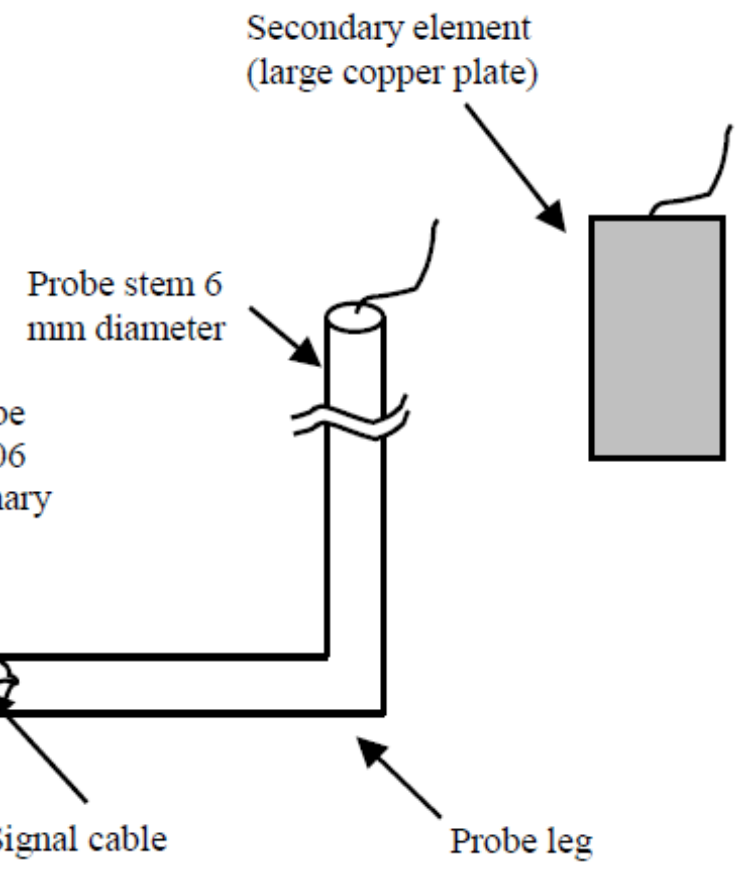

Fig. 2. Schematic of the phase detection probe. 


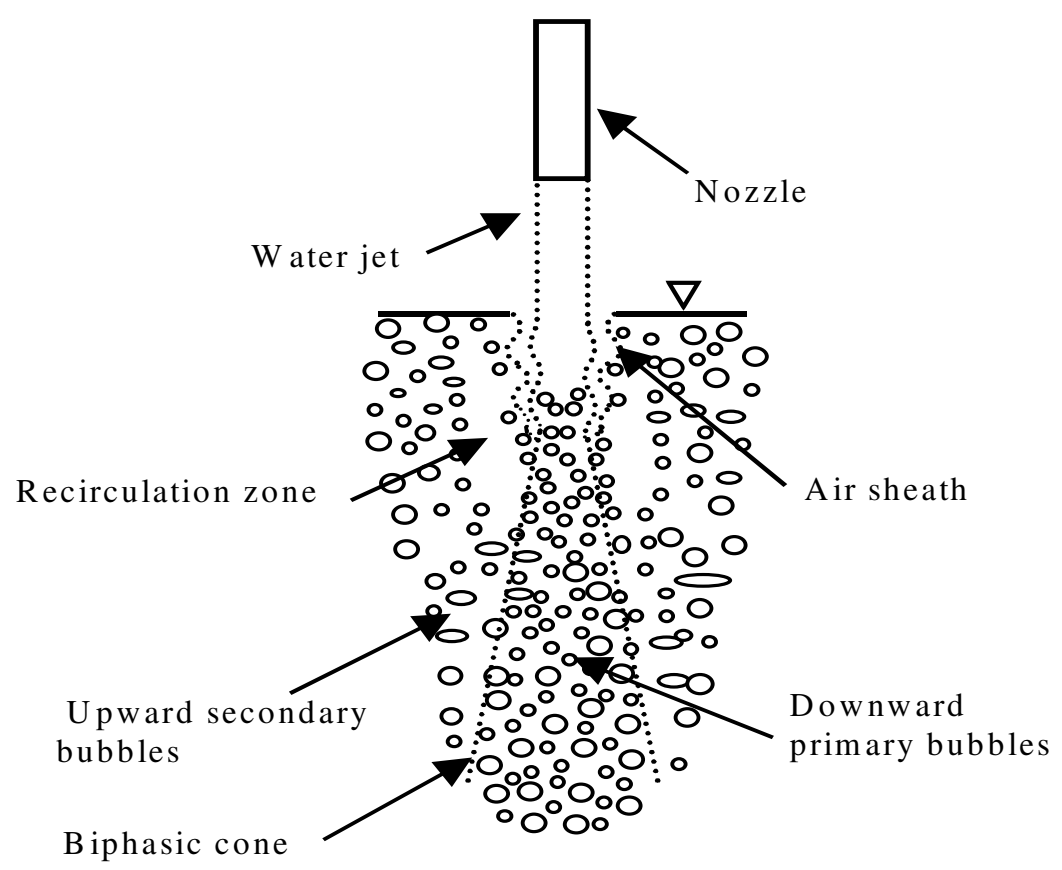

Fig. 3. Schematic representation of bubble formation due to liquid jet impingement (Reproduced from [14]).

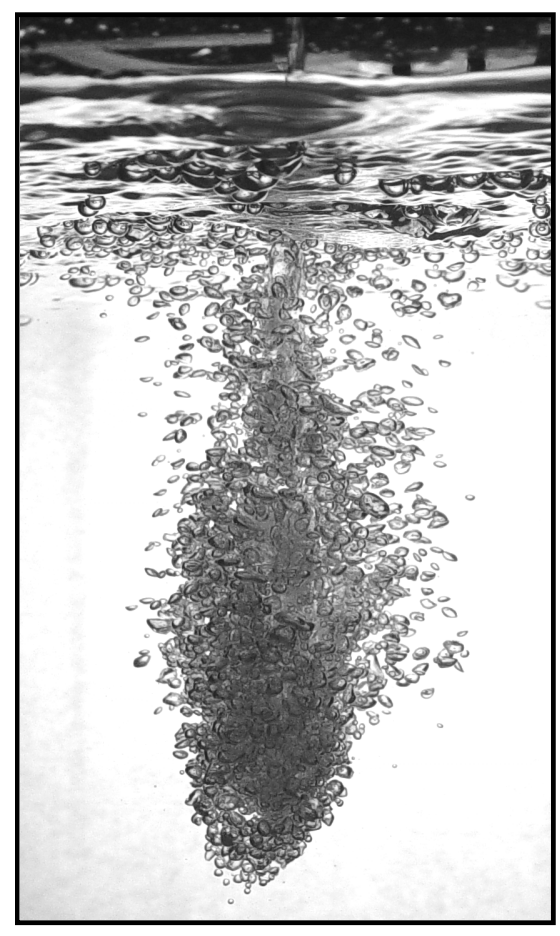

Fig.4. Shape of biphasic zone (Photograph Reproduced from [14]) 


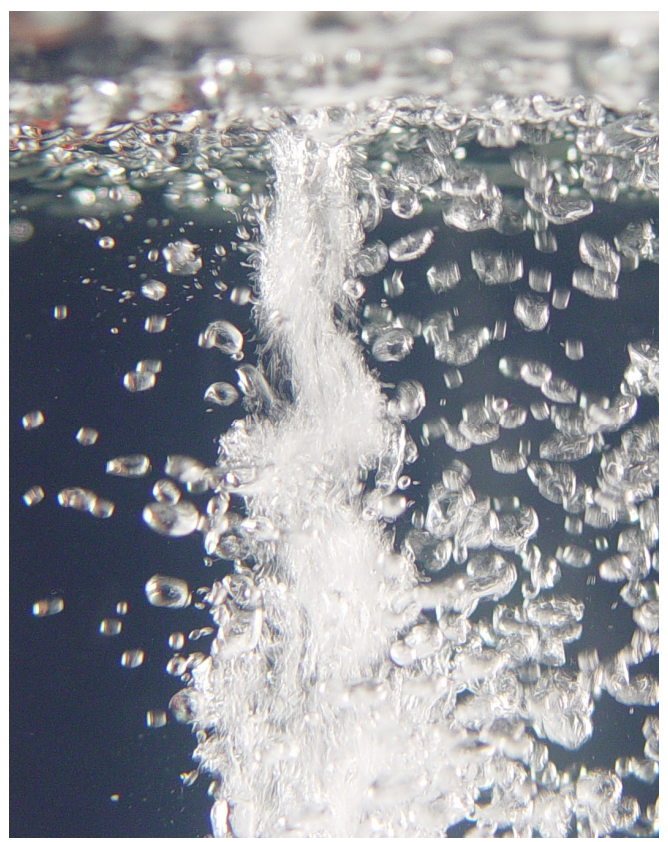

Fig.5. Vortex formation due to plunging liquid jet (Photograph Reproduced from [14])

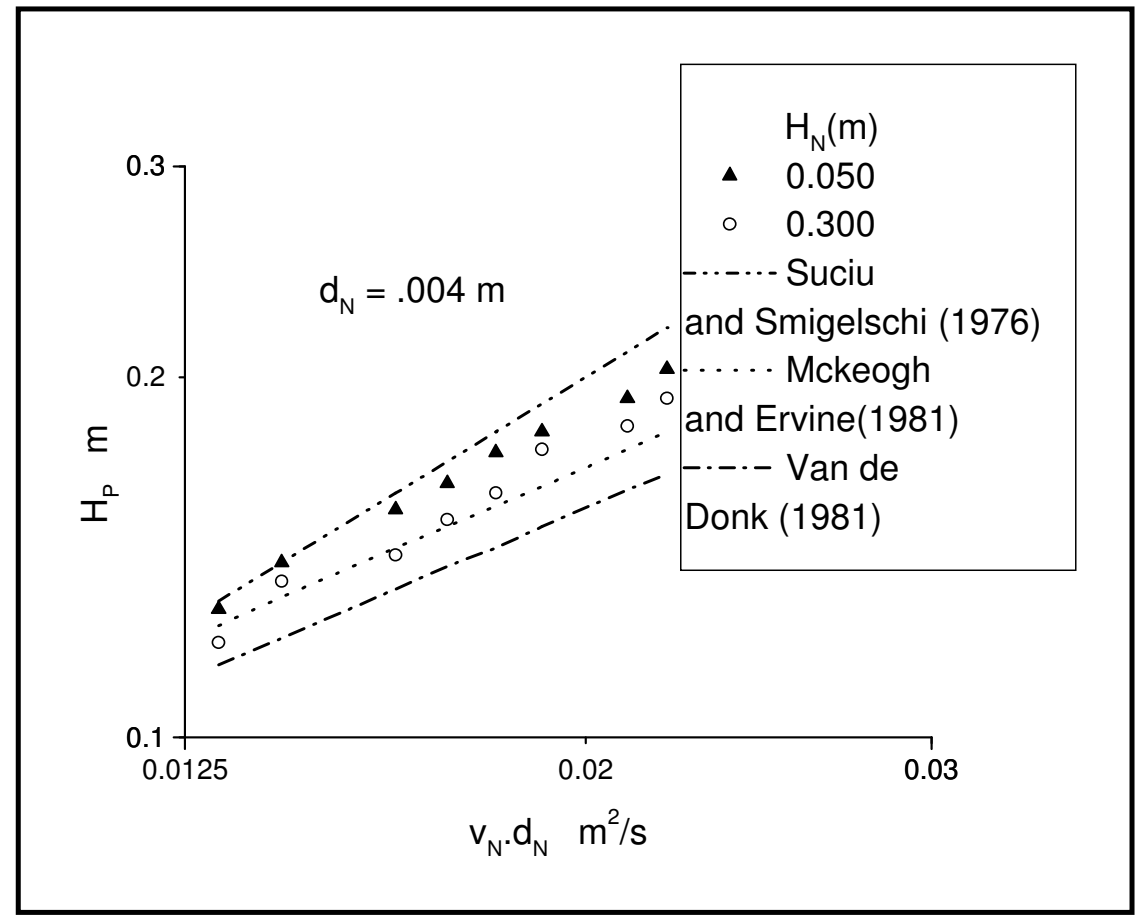

Fig. 6. Variation of bubble penetration depth with jet momentum (4 mm nozzle). 


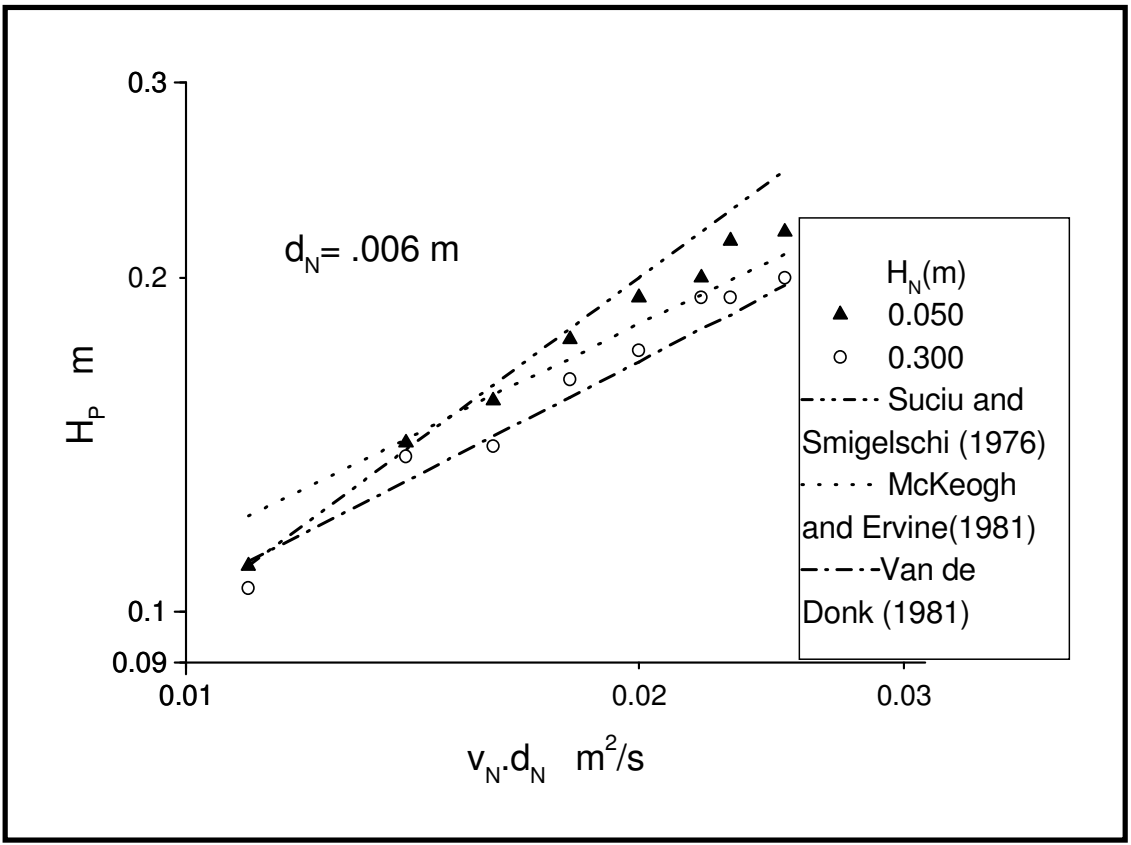

Fig. 7. Variation of bubble penetration depth with jet momentum (6 mm nozzle).

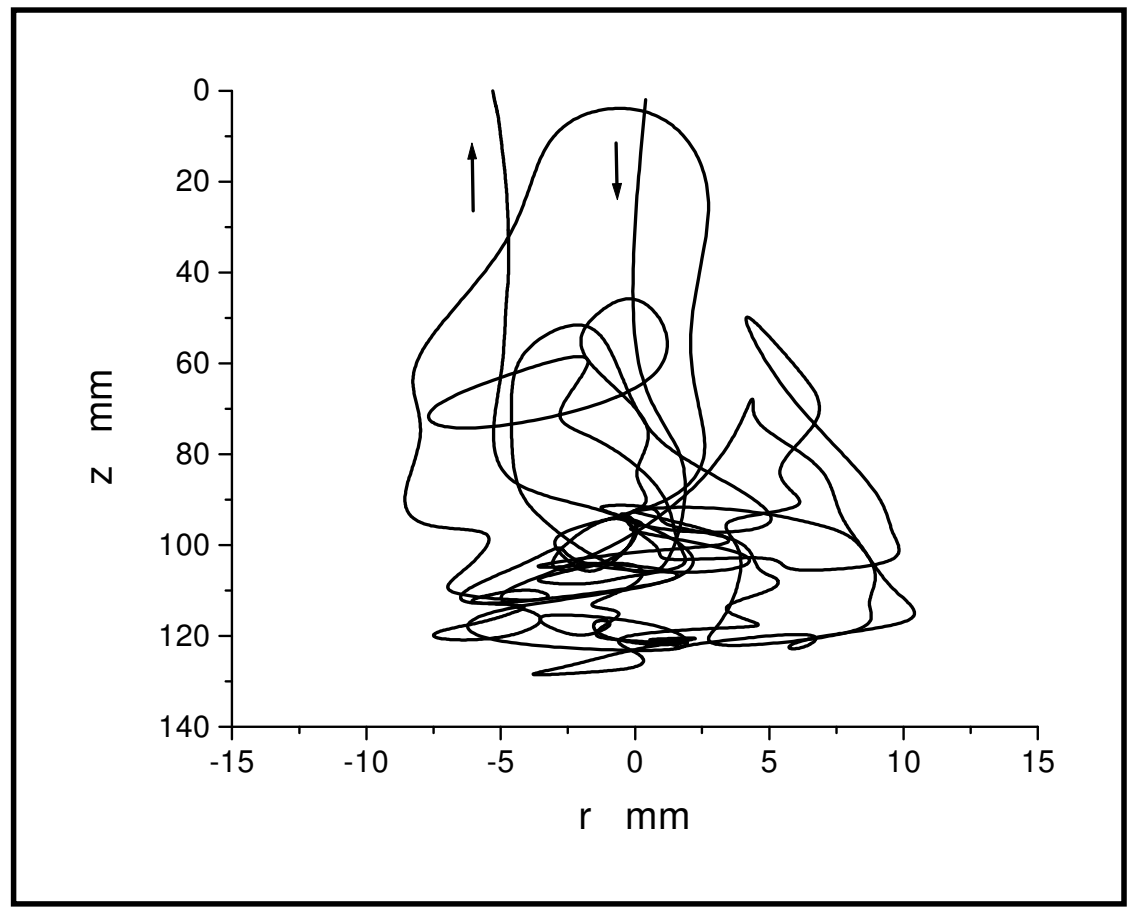

Fig. 8. Trajectory of a single entrained bubble ((Reproduced from [14]). 

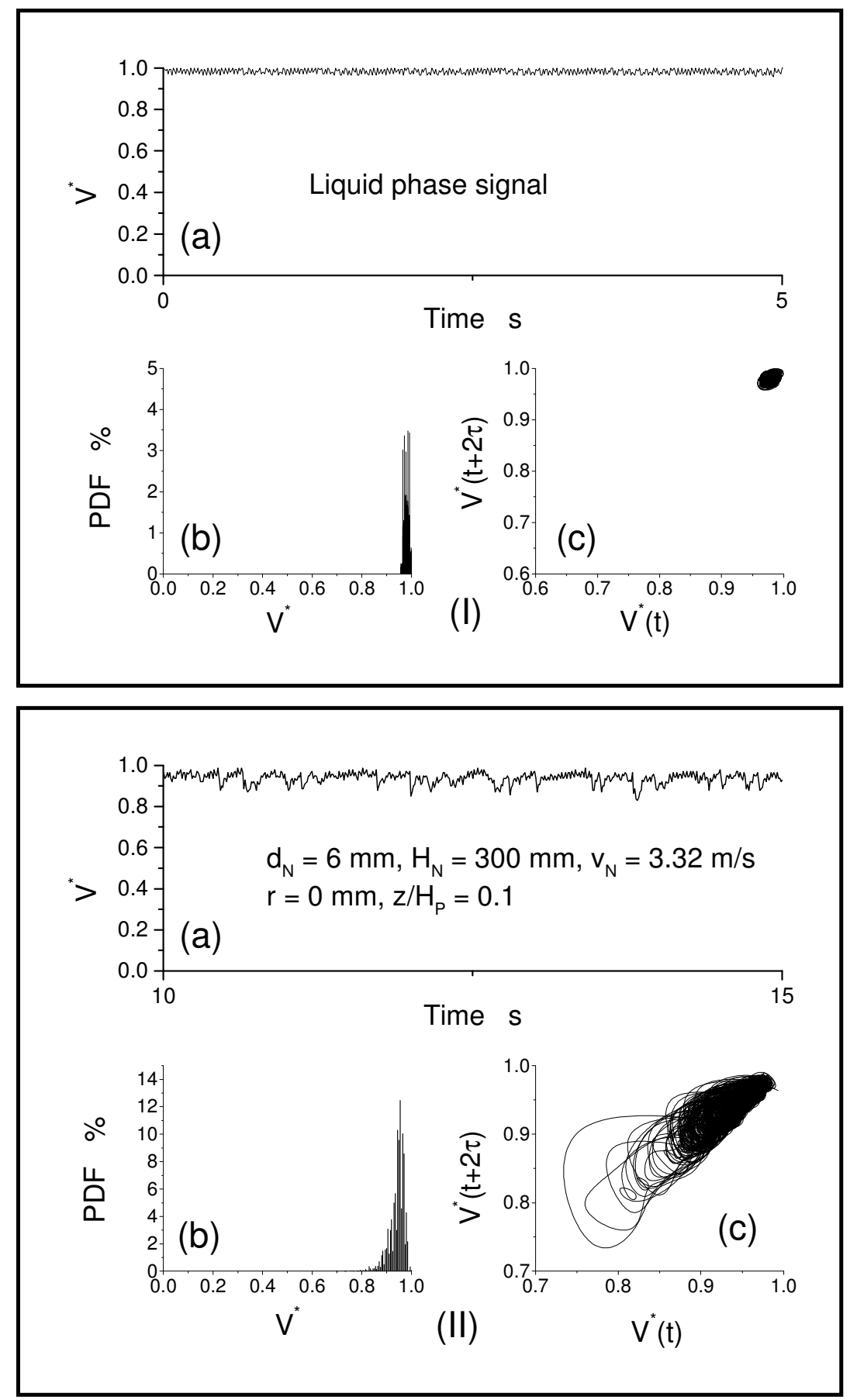

Figs. 9.(I-II). Probe signals at different axial positions (I) Liquid phase signal, (II) $\mathrm{z} / \mathrm{H}_{\mathrm{P}}=0.1$. 

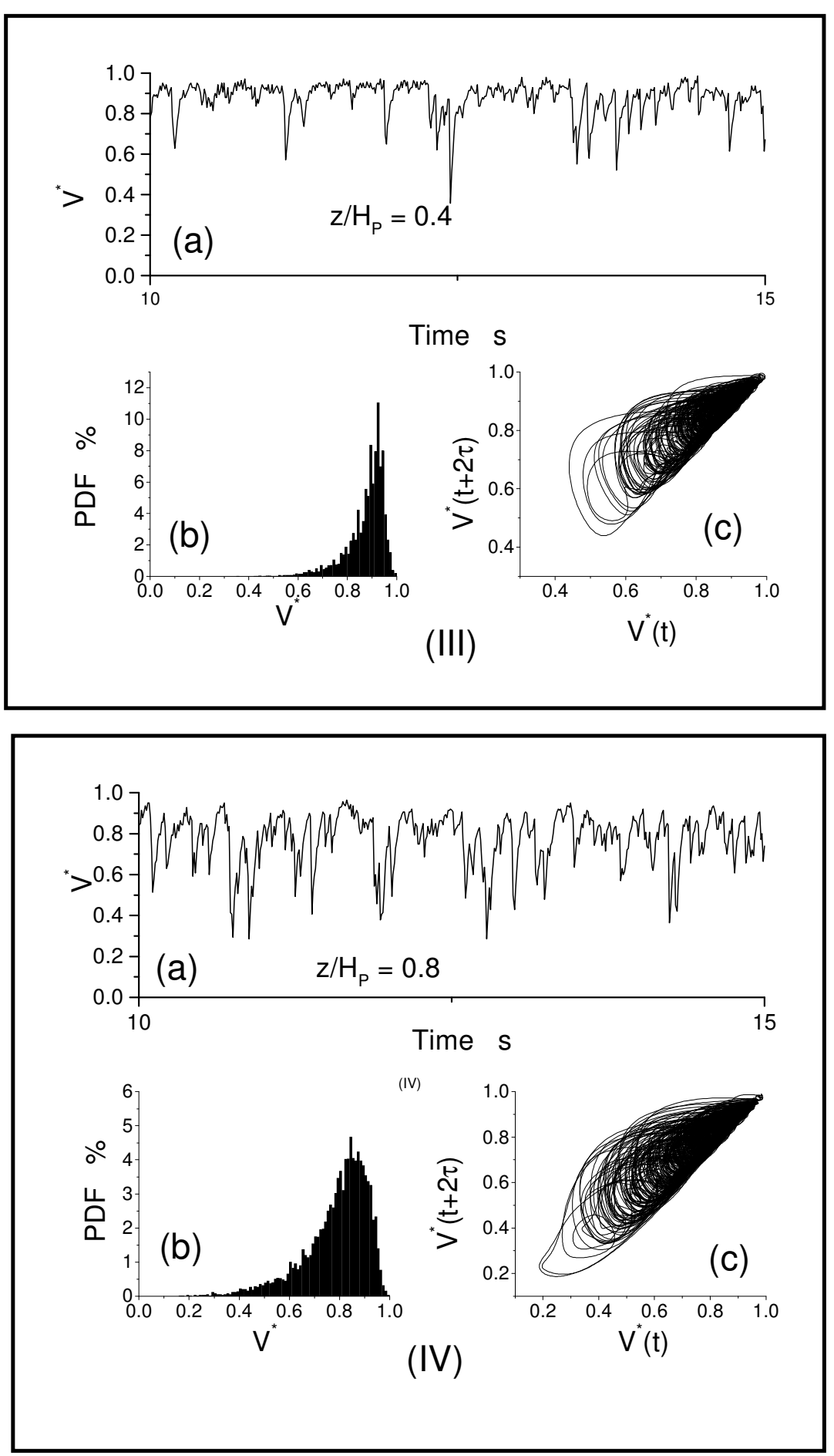

Figs. 9.(III-IV). Probe signals at different axial positions (III) $\mathrm{z} / \mathrm{H}_{\mathrm{P}}=0.4$, (IV) $\mathrm{z} / \mathrm{H}_{\mathrm{P}}=0.8$. 

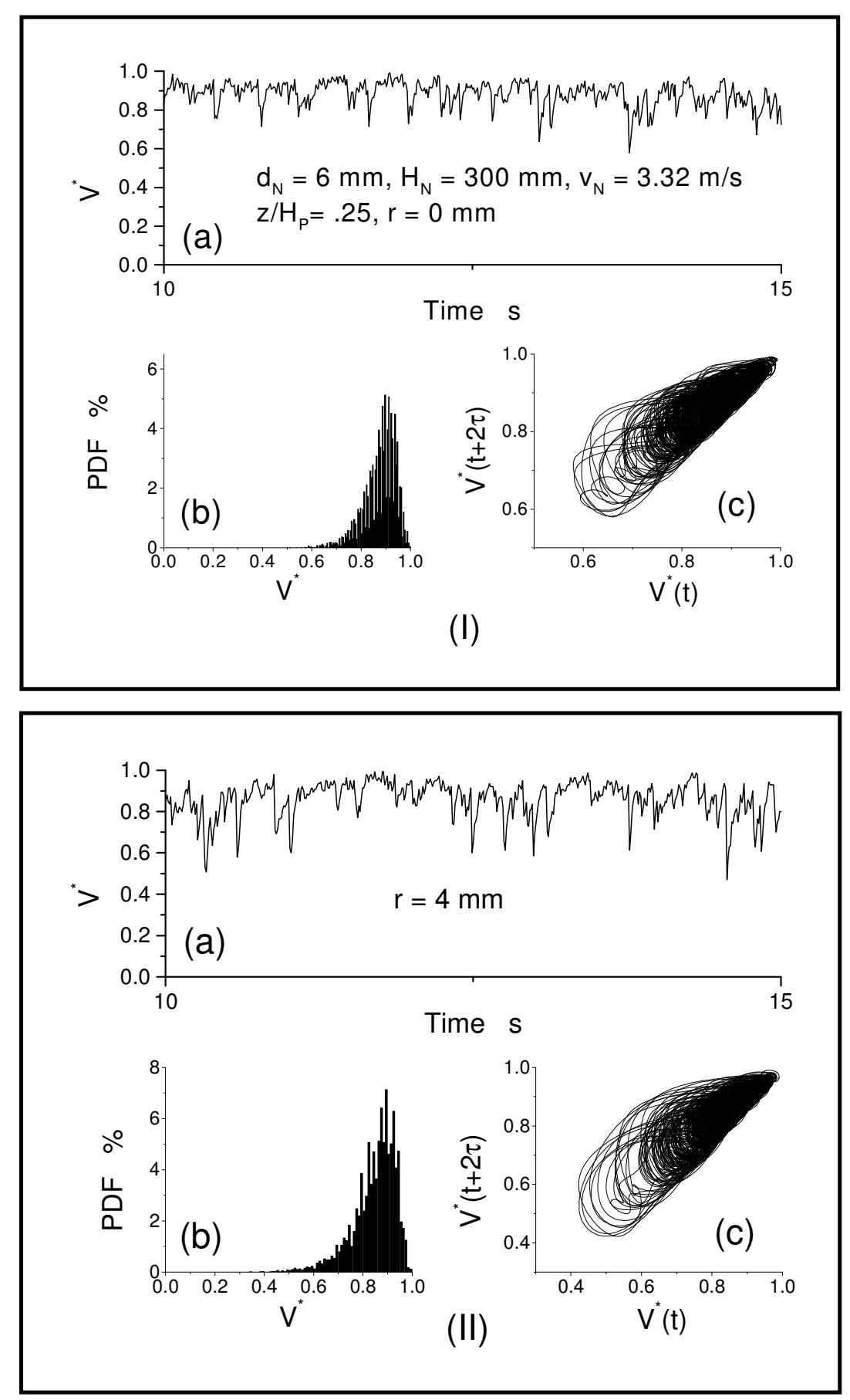

Figs. 10(I-II). Probe signals along radial direction (I) $r=0.0 \mathrm{~mm}$, (II) $r=4.0 \mathrm{~mm}$. 

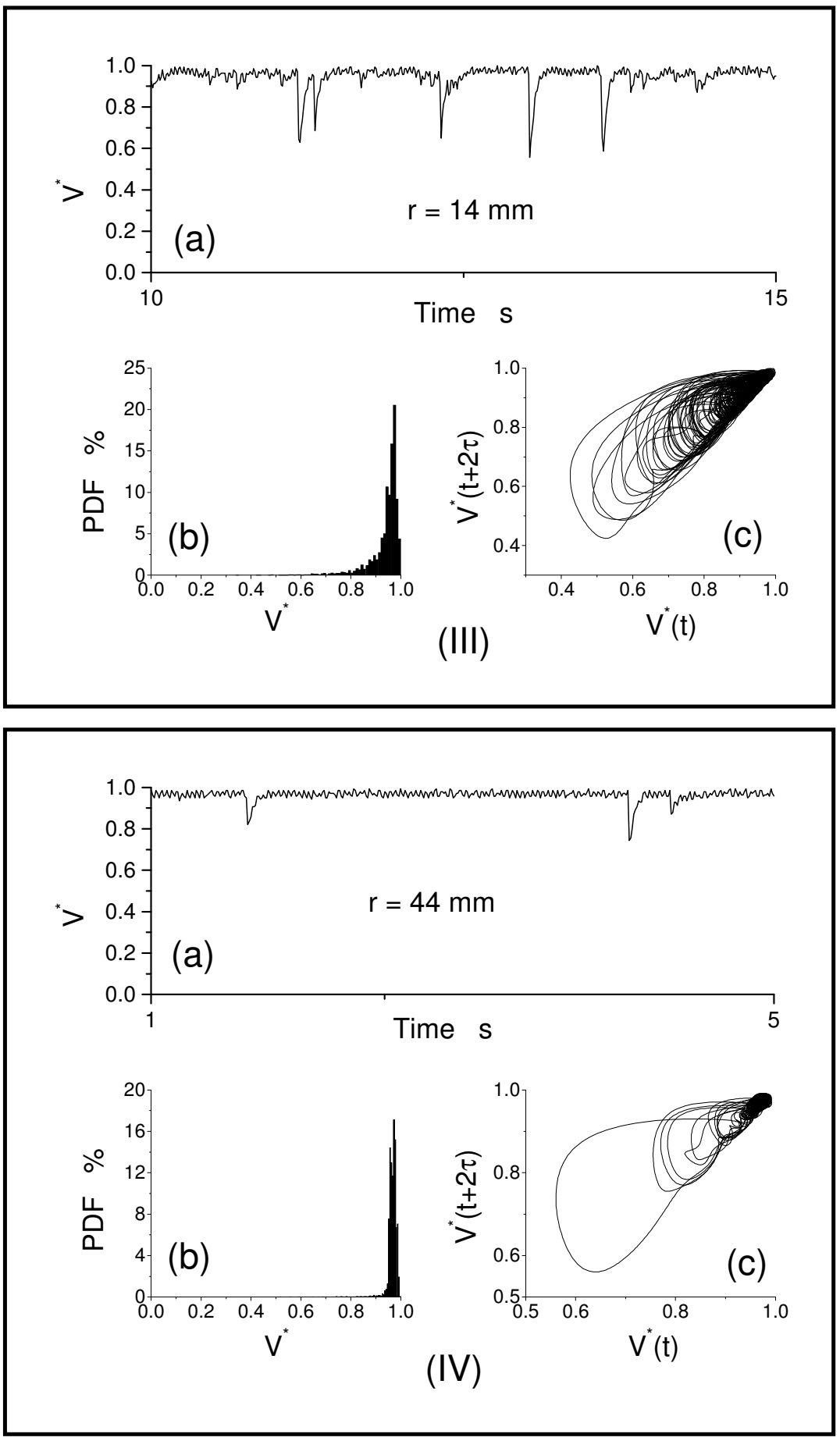

Figs. 10(III-IV). Probe signals along radial direction (III) $r=14.0 \mathrm{~mm}$, (IV) $r=44.0 \mathrm{~mm}$. 


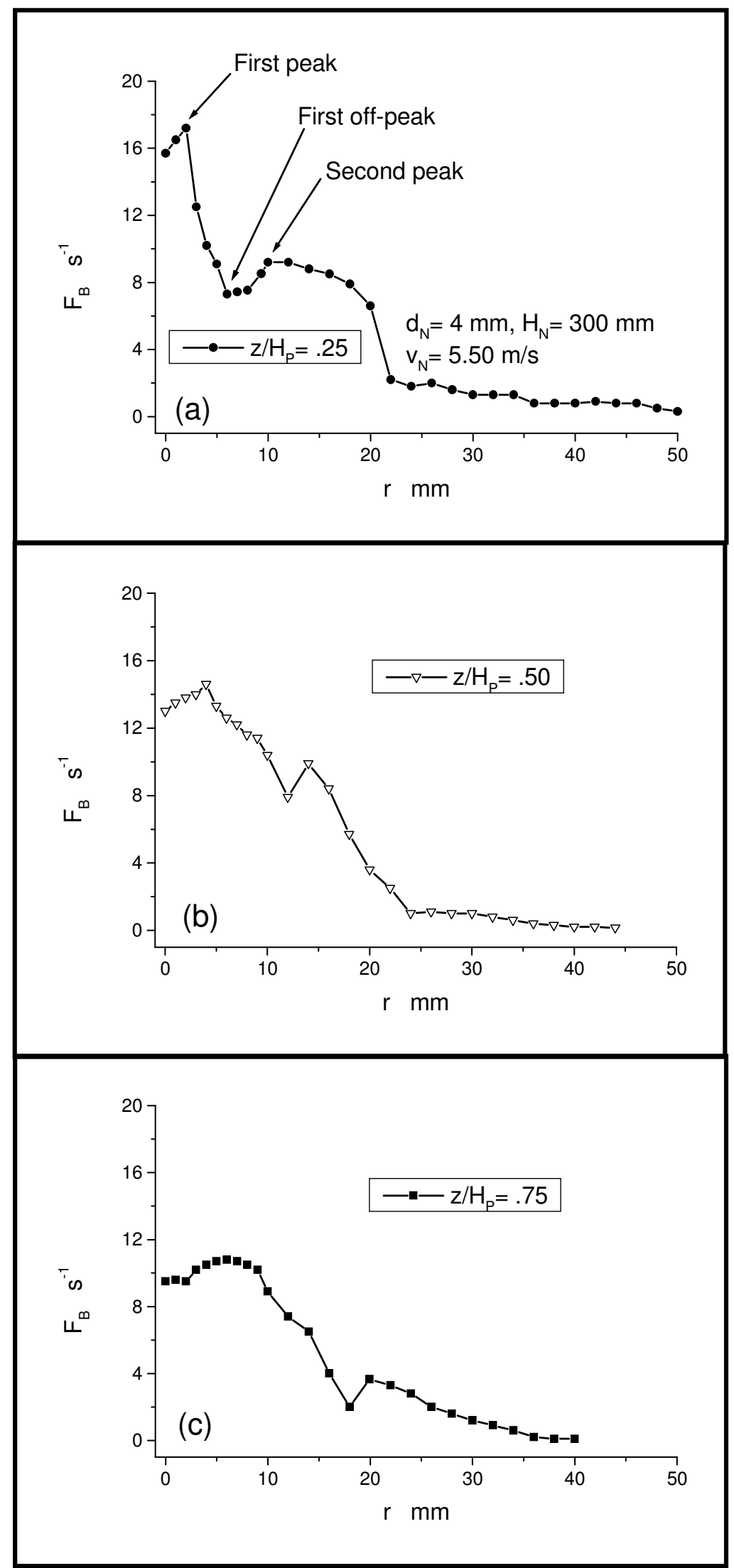

Figs. 11(a-c). Variation of bubble count rate in radial direction at $\mathrm{v}_{\mathrm{N}}=5.50 \mathrm{~m} / \mathrm{s}$ (a) $\mathrm{z} / \mathrm{H}_{\mathrm{P}}=0.25$, (b) $\mathrm{z} / \mathrm{H}_{\mathrm{P}}=0.50$, (c) $\mathrm{z} / \mathrm{H}_{\mathrm{P}}=0.75$. 


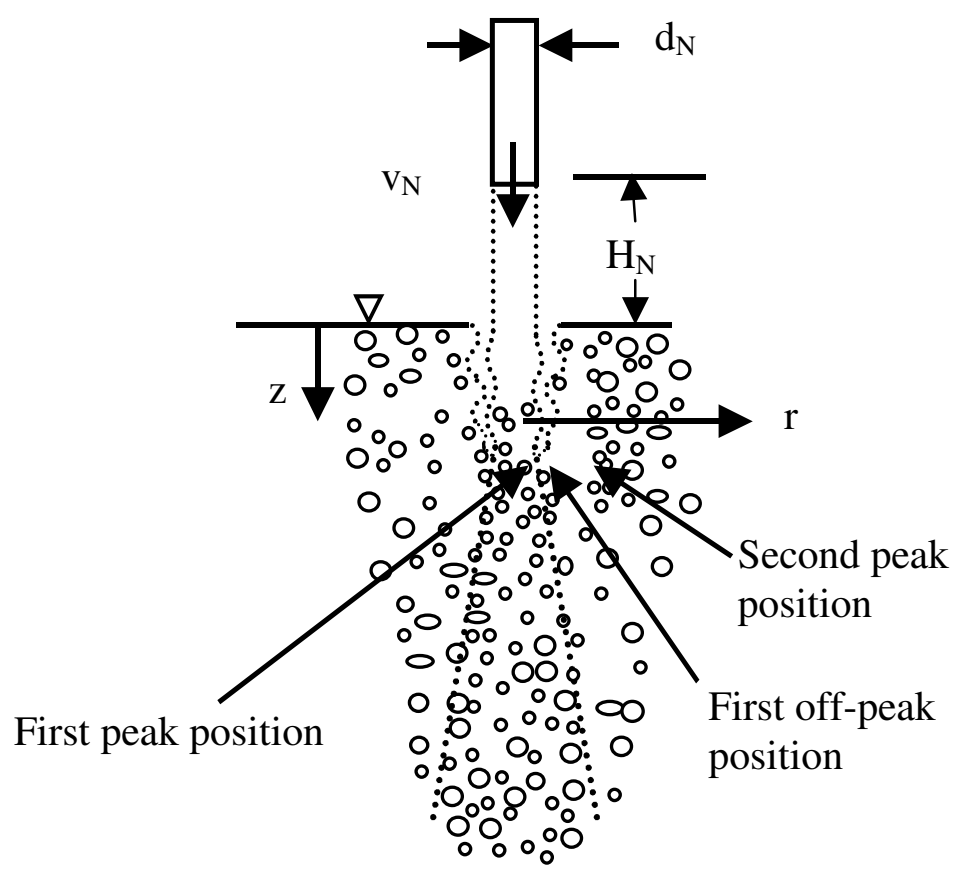

Fig.12. Schematic representation of peak and off-peak positions.

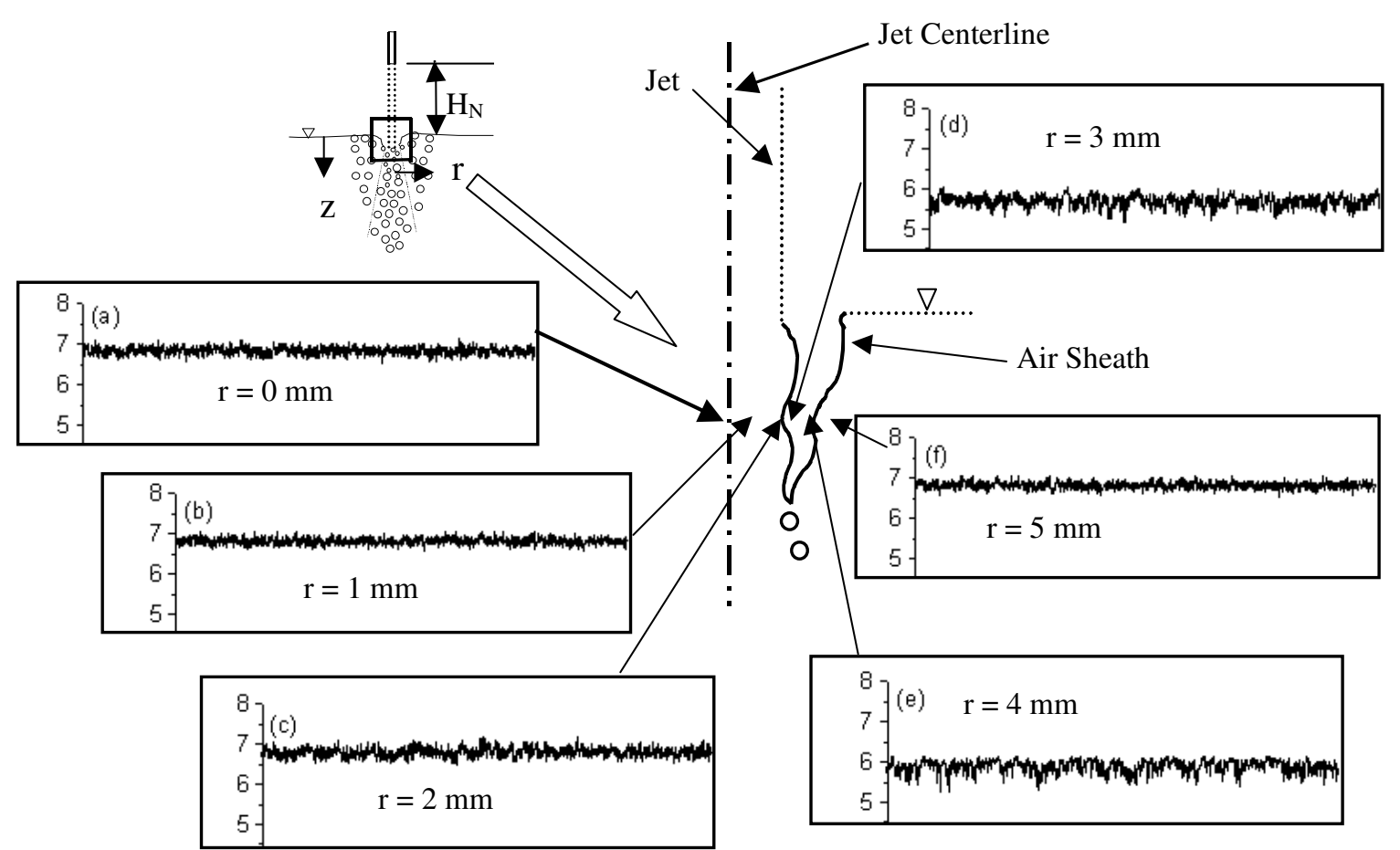

Fig. 13. Probe signals within and around the air sheath $\left(H_{N}=300 \mathrm{~mm}, v_{N}=5.50 \mathrm{~m} / \mathrm{s}, d_{N}=4 \mathrm{~mm}, \mathrm{z}=8 \mathrm{~mm}\right)$. 
Table 1. The Range of Experimentation

\begin{tabular}{|c|c|c|c|}
\hline $\begin{array}{c}\text { Nozzle } \\
\text { diameter } \\
\mathrm{d}_{\mathrm{N}}(\mathrm{mm})\end{array}$ & $\begin{array}{c}\text { Jet velocity } \\
\mathrm{v}_{\mathrm{N}}(\mathrm{m} / \mathrm{s})\end{array}$ & $\begin{array}{c}\text { Nozzle } \\
\text { height } \\
\mathrm{H}_{\mathrm{N}}(\mathrm{mm})\end{array}$ & $\begin{array}{c}\text { Nozzle } \\
\text { geometry } \\
\mathrm{L}_{\mathrm{N}} / \mathrm{d}_{\mathrm{N}}\end{array}$ \\
\hline 4 & $3.25-5.5$ & 50,300 & 25 \\
6 & $2.23-4.18$ & 50,300 & 25 \\
\hline
\end{tabular}

Table 2. Inception velocities $\left(\mathrm{v}_{\mathrm{C}}\right)$

\begin{tabular}{|c|c|c|}
\hline $\begin{array}{c}\text { Nozzle diameter } \\
\mathrm{d}_{\mathrm{N}}(\mathrm{mm})\end{array}$ & $\begin{array}{c}\text { Nozzle height } \\
\mathrm{H}_{\mathrm{N}}(\mathrm{mm})\end{array}$ & $\begin{array}{c}\mathrm{v}_{\mathrm{C}} \\
(\mathrm{m} / \mathrm{s})\end{array}$ \\
\hline 4 and 6 & 50 & 1.6 \\
4 and 6 & 300 & 1.2 \\
\hline
\end{tabular}

